\title{
Mouse Models of Experimental Vascularized Composite Allotransplantation
}

\author{
Zhanzhuo Li • Alain Le Moine
}

Published online: 18 June 2014

(C) Springer International Publishing AG 2014

\begin{abstract}
The clinical practice of vascularized composite allotransplantation (VCA) has been limited by the potential side effects of chronic immunosuppression. Studies using rodent models have been useful for dissecting mechanisms underlying immunological events induced by VCA and for developing protocols such as mixed chimerism for tolerance induction. Mouse models of VCA have great advantages over rat and other rodents with regard to dissection of immunologic mechanisms; however, the microsurgical revascularization procedures that are required are much more difficult. Here we review recent advances in surgical and immunological methods for successful VCA in the mouse model.
\end{abstract}

Keywords Mouse $\cdot$ Models $\cdot$ Microsurgery $\cdot$ Mixed chimerism $\cdot$ Tolerance $\cdot$ Vascularized composite allotransplantation

\section{Introduction}

Vascularized composite allotransplantation (VCA) has gained increasing importance in clinical practice, which may evolve into an important component of multidisciplinary approaches to post-injury reconstruction. VCA transplants contain multiple tissues including skin, muscle, tendon, nerve, and bone, and each individual tissue component possesses unique immunologic characteristics that collectively contribute to the

Z. Li $\cdot$ A. Le Moine

Institute for Medical Immunology (IMI), Universite Libre de

Bruxelles (ULB), Brussel, Belgium

\section{Z. $\operatorname{Li}(\bowtie)$}

Laboratory of Molecular Immunology, National Institute of Allergy and Infectious Diseases (NIAID), National Institutes of Health (NIH), Bethesda, MD, USA

e-mail: zhanzhuo@niaid.nih.gov immunologic response to VCA [1]. Experimental transplantation models in rodents are critically important in dissecting mechanisms underlying the alloimmune response and for ultimately developing tolerance-inducing strategies, including in VCA.

The first experimental VCA in rodents was reported by Schwind in 1936 [2], decades before the first clinical VCA attempt in humans [2]. This study, which involved heterotopic hindlimb transplantation in the rat using parabiosis, not only established the microsurgical feasibility of the model, but also addressed the central issue in transplantation, preventing rejection and inducing tolerance. Since the late 1970s, extensive studies have been performed using the rat model [3], and today this model remains the main experimental rodent VCA model. Ever since introduction of mouse VCA models in 1998, the number of VCA studies reported in rats is nearly double the number of those in mice.

Nevertheless, to address immunologic mechanisms mouse models have several advantages: better defined genetics, extensive availability of congenic, transgenic, and knockout strains, and immunological/molecular biological reagents, as well as comparatively low capital and housing costs, about one-third the cost for rats [4]. The major obstacle to wider use of the mouse VCA model is the difficulty of microsurgical manipulation of murine femoral vessels [5]. These vessels are very small (diameter $0.2-0.4 \mathrm{~mm}$ in the artery and $0.6-0.75 \mathrm{~mm}$ in the vein at the level of mid-thigh) and very delicate (the wall of the femoral vein has only a few layers of cells) $[6,7 \bullet \bullet, 8]$. Despite this, there have been 12 models of murine VCA described since its introduction more than 15 years ago, including transplantation models involving hindlimb, osteomyocutaneous (OMC) flap, ear, face, and larynx, in 23 publications from the literature in English (Table 1).

Here we provide the first review of these models, focusing on both advances in microsurgical technique and insights into immunoregulation of allotransplantation. 
Table 1 Models of Murine Vascularized Composite Allotransplantation

\begin{tabular}{|c|c|c|c|c|c|c|}
\hline Author & Year & VCA graft & Ortho/Hetero & Vascular Anastomosis & Ischemia Time & Success Rate \\
\hline Cooley et al [9] & 1998 & Groin flap & Hetero & End-to-side & NS & $75 \%$ \\
\hline Tung et al [8] & 2001 & & & $\begin{array}{l}\text { A: end-to-end } \\
\text { V: end-to-side }\end{array}$ & $45-60 \min$ & $75 \%$ \\
\hline Ulusal et al [11] & 2005 & & & & $60 \mathrm{~min}$ & $90 \%$ \\
\hline Jiang et al [10] & 1998 & Ear & Ortho & $\begin{array}{l}\text { A: end-to-end } \\
\text { V: end-to-side }\end{array}$ & $45 \pm 5 \min$ & $94 \%$ \\
\hline Zhang et al [12] & 1999 & Limb & Ortho & A \& V: end-to-end & $<1.5 \mathrm{~h}$ & $83 \%$ \\
\hline Tung et al [8] & 2001 & Limb & Hetero & $\begin{array}{l}\text { A: end-to-end } \\
\text { V: end-to-side }\end{array}$ & $45-60 \min$ & $70 \%$ \\
\hline $\begin{array}{l}\text { Tung et al } \\
\quad[16,30,25,28,27,26,17]\end{array}$ & $2001,02,03,05,05,05,08$ & OMC flap & Hetero & $\begin{array}{l}\text { A: end-to-end } \\
\text { V: end-to-side }\end{array}$ & $45-60 \min$ & $73 \%$ \\
\hline Cohen et al [29] & 2006 & & & & NS & NS \\
\hline Yan et al [18] & 2011 & & & & $60 \mathrm{~min}$ & $69.3 \%$ \\
\hline Foster et al [13] & 2003 & Limb & Ortho & $\begin{array}{l}\text { A: end-to-end } \\
\text { V: cuff }\end{array}$ & $56 \mathrm{~min}$ & $73 \%$ \\
\hline Zhong et al [15] & 2007 & Limb & Ortho/Hetero & $\begin{array}{l}\text { A: end-to-end } \\
\text { V: end-to-side }\end{array}$ & NS & $86 \%$ \\
\hline Li et al $[19,31]$ & 2007,08 & OMC flap & Hetero & A \& V: end-to-side & $40 \mathrm{~min}$ & $87.5 \%$ \\
\hline Shipchandler et al [23] & 2009 & Larynx & Hetero & A \& V: end-to-side & NS & NS \\
\hline Sucher et al $[7 \bullet \bullet]$ & 2010 & Limb & Ortho & A \& V: cuff & NS & $62 \%$ \\
\hline Sucher et al [22] & 2012 & Face & Ortho & A \& V: cuff & NS & $78 \%$ \\
\hline Sucher et al $[7 \bullet \bullet]$ & 2010 & OMC flap & Hetero & A \& V: cuff & NS & $90 \%$ \\
\hline Lin et al $[6,21 \bullet]$ & 2013,14 & & & & NS & $85 \%$ \\
\hline
\end{tabular}

Hetero, heterotopic

Ortho, orthotopic

$N S$, not specified

\section{Mouse VCA Models}

The first two reports of murine VCA were for a vascularized groin skin flap transplant and an ear transplant, both published in $1998[9,10]$. In the former study, Cooley and colleagues isolated an epigastric groin skin flap based on a pedicle composed of inferior epigastric-femoral vessels, and heterotopically transplanted it to the recipient groin fossa [9]. This study is an important landmark for microvascular surgery involving mouse femoral vessels, and provided a foundation for future advances in the field. The method involved use of 11-0 sutures on a 50-micron needle with 40x magnification, and concluded that end-to-side anastomosis was optimal. In 2001 and 2005, Tung et al [8] and Ulusal et al [11] described similar groin flap models, with some modifications. In Jiang and colleagues' orthotopic ear transplant model [10], the entire ear was used as the graft with all-level branches of the common carotid artery and jugular vein supplying the ear as the pedicle. This study expanded our knowledge of murine VCA, indicating that blood vessels in the neck can be a good alternative to the femoral vessels, with a high success rate $(94 \%)$ for anastomosis (Table 1$)$.
Zhang et al published a murine hindlimb transplantation model in 1999 [12], the first successful limb transplantation model in mice, and a translation of the standard rat hindlimb transplantation model described by Doi or Shapiro [2]. The model consisted of orthotopic transplantation of an intact mouse hindlimb at mid-femur level, with microsurgical repair of the femoral vessels with 11-0 sutures; nerve repair was not performed. The procedure took $2 \mathrm{~h}$; ischemia was less than $1.5 \mathrm{~h}$. The authors achieved a 10 out of 12 success rate in the first $24 \mathrm{~h}$ post-operatively, which they attributed to several factors: adequate trimming of the adventitia, especially at the vein; appropriate vascular anastomotic tension by maximizing the length of both the donor and recipient vascular stump with a shortened bone stump; and sequenced artery-then-vein microvascular repair; post-operative fluid replacement was also critical to animal survival. In 2003, Foster et al published a very similar murine orthotopic limb transplantation model [13]. The most prominent modification in this model was in the venous anastomosis, in which the investigators used the "cuff" technique, first introduced in late 1970s [14], to simplify the anastomotic procedure. According to the authors, the total procedure and ischemia times were successfully reduced; however, the success rate $(73 \%, 11$ out of 15$)$ was not 
significantly improved even though they used large-sized recipient mice (body weight 35 - $50 \mathrm{~g}$ ). More recently, Zhong et al [15] \& Sucher et al [7••] published their orthotopic limb transplantation models, respectively. In Zhong's model, the artery was repaired in an end-to-end pattern and the vein was repaired in an end-to-side pattern using 11-0 sutures. The success rate was 20 out of 23 . Sciatic nerve repair was also performed, although no nerve regeneration or functional recovery study was noted in the article [15]. In Sucher's model, both femoral vessels were repaired using the "cuff" technique. There was moderate reduction of the recipient procedure time with this simplified microvascular technique; however, the success rate was $62 \%$, not as high as compared to models using sutures. The authors also repaired the sciatic nerve with 11-0 suture, and performed several functional recovery studies $[7 \bullet \bullet]$.

In addition to the orthotopic limb transplantation models, there have been attempts made in the area of heterotopic transplantation. The advantage of this approach is obvious: it is far less traumatic since it avoids recipient limb amputation, thereby reducing blood loss, surgery length and physiological stress in the recipient mouse. Tung et al published the first successful heterotopic murine limb transplantation model in 2001 [8], grafting a distal hindlimb at knee level, based on a pedicle composed of femoral vessels. The graft pedicle was heterotopically anastomosed to recipient femoral vessels at the upper thigh. The venous anastomosis was performed first in an end-to-side pattern, followed by end-to-end arterial anastomosis, with no nerve repair. The graft was placed on the back of the recipient mouse near the tail base to minimize the risk of autotomy, a common complication seen in mice receiving non-reinnervated limb grafts. The team later published a modified heterotopic model [16, 17], in which a tibia-based OMC flap was transplanted as the graft, in the same pattern as that of the distal limb graft published earlier. The success rate in both models was $72-73 \%$. The authors also reported their experience with microvascular vein manipulation, a technique they named "sewing underwater," to improve and maintain visualization of the lumen of the venotomized femoral vein by intermittent irrigation with heparinized saline. The key was to keep the lumen open by keeping the vein submerged, which improved visualization and manipulation accuracy of the very delicate, transparent venous wall. The team's latest study with the heterotopic model was in 2011, in which the most prominent modification was the rejoining of recipient sciatic nerve with the limb allograft counterpart. Microscopic analysis of post-allotransplantation nerve regeneration was also performed [18].

Our own heterotopic OMC flap model was first published in 2007 [19], in which we made five major modifications to previously published models. First, we developed a femurbased OMC flap. Compared to the distal limb, the femurassociated vasculature has a larger diameter and is, therefore, less likely to develop thromboembolic events. Second, the donor femur was released intact by disarticulation of hip and knee joints to maximally preserve the proliferation potential of donor bone marrow. Third, for the muscle attached to the femur, the outer layer was removed to reduce the graft size, and the inner layer was preserved to protect the associated bone-supplying microvasculature (Fig. 1a). Fourth, to maximize the vascular pedicle length for appropriate anastomotic tension, we developed a pedicle based on femoral, iliac and aortic/IVC vessels. To do this, the dissection was started from the external iliac vessels after severing the inguinal ligament, the main trunk of iliac vessels were followed cephalad along the abdominal aorta/IVC to the level of the renal vessels, and all the branches along the way were ligated individually with 10-0 or 5-0 suture depending on the size, including the ipsilateral internal iliac vessels and contralateral common iliac vessels (Fig. 1a). Then the overlying fascia and vascular
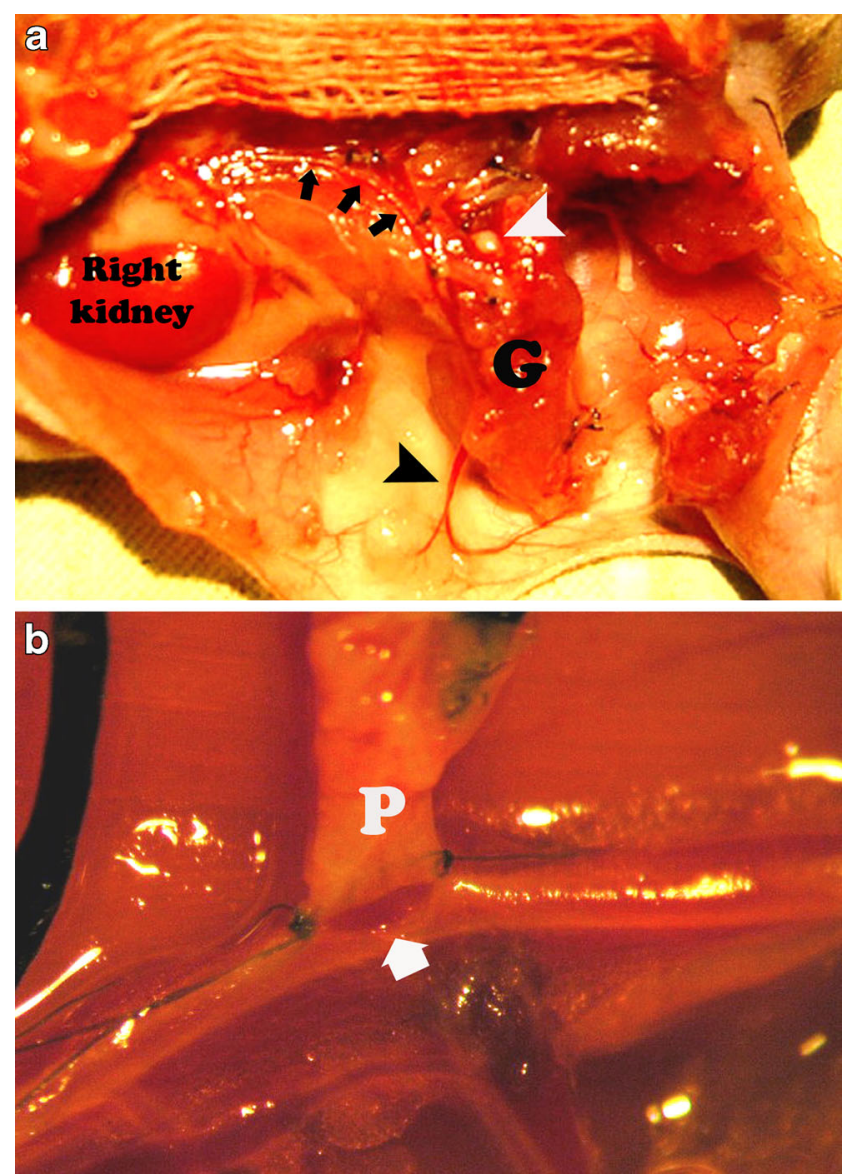

Fig. 1 Surgical procedures used in murine VCA models. (a): Limb graft (G) created after hip \& knee joint disarticulation. The vascular pedicle (arrows) was not transected until the recipient vessels were ready for undergoing vascular surgery. White arrow head indicates exposed femur head after hip joint disarticulation, and black arrow head indicates the vessel branch supplying the skin component of the OMC flap. (b): A longer-than-usual arteriotomy/venotomy (white arrowhead) was created at recipient femoral vessels approximating the caliber of the graft's vascular pedicle $(\mathrm{P})$ 
sheath covering the abdominal aorta/inferior vena cava (IVC) was dissected and the vessels freed from each other before they were transected by a microdissection scissor [20]. This approach is somewhat similar to the pedicle development described by Jiang et al in their ear model. The time needed for donor surgery was about 1.5 to $2 \mathrm{~h}$. Fifth, we used end-toside anastomosis in both artery and vein as this approach is more permissive in cases of a large discrepancy in vascular caliber. A longer-than-usual arteriotomy/venotomy approximating the caliber of the aorta/IVC was performed at the recipient femoral vessels (Fig. 1b), and then the anastomosis was performed in a continuous pattern. Ten to 14 stitches are necessary to finish the procedure. It is essential before placing the anchor stitches that the vessels are thoroughly irrigated with heparinized, cold saline using a 26-gauge cannula connected to a 1-ml syringe to dislodge the adherent clots. Repeated, intermittent irrigation was performed in a similar way throughout the anastomosis, which is important for keeping the lumen patent and improving visualization of the vessel walls. Adequate visualization of the lumen minimized the need for direct vessel wall manipulation by surgical instruments, thereby minimizing the risk of endothelium activation/ thrombosis, especially in the extremely delicate femoral vein. The overall success rate in our model was $87.5 \%$; the time needed for recipient surgery was about $1 \mathrm{~h}$. To reduce ischemia time, the recipient surgery was started before graft vascular pedicle transection (Fig. 1a); the graft ischemia time was $\sim 40 \mathrm{~min}$. Minimal bleeding occurred during the procedure and the animals were doing well postoperatively with no autotomy observed.

In the same publication that described their orthotopic model, Sucher et al also introduced their heterotopic OMC flap model $[7 \cdot \bullet]$. The novelty of this model is the grafting of a femur-based OMC flap to the cervical area using the "cuff" technique, an approach more commonly employed in the rodent heterotopic heart transplantation model. With this modification, the authors achieved shorter procedure duration, less bleeding/invasiveness, and a much higher success rate $(85-$ $90 \%$ ). In a more recent study from the same team, a viable graft was observed in some animals at 180 days after transplantation [21•], and histological study revealed good longterm patency of the blood vessels inside the cuff [6]. The very same technique was also employed in a murine hemiface allotransplantation model developed by the team [22]. In this model, a vascular pedicle composed of common carotid artery and external jugular vein was reunited with the recipient counterparts to achieve graft revascularization, and the initial success rate was promising [22].

Another cervical heterotopic transplantation model in mice is the heterotopic laryngeal transplantation model by Shipchandler et al [23]. The authors developed a laryngeal graft based on a vascular pedicle composed of bilateral common carotid artery and its thyroid branches. The graft revascularization was achieved via end-to-side anastomosis of the pedicle vessels to recipient left common carotid artery and internal jugular vein, respectively. The authors reported good vascular patency at day 30 post-transplant after experiencing several unsuccessful cases at the beginning [23].

\section{Immune Modulation in Murine VCA Studies}

The fundamental dilemma in VCA is that the medical condition undergoing treatment, while disfiguring, is not by itself life-threatening, whereas the treatment must be sustained by potentially toxic immunosuppression similar to that for solid organ grafts. Therefore, a major challenge in the field is to develop a non-toxic immunomodulatory strategy, such as mixed chimerism, for donor-specific transplant tolerance. So far, mixed chimerism has not been achieved in the clinic despite extensive experimental studies, mainly in rats. The introduction of murine VCA models has expanded the opportunity for progress in this field (Table 2). Extensive studies have been performed regarding the mechanisms of tolerance in murine mixed chimerism models in transplantation contexts other than VCA. It has been established that central clonal deletion of alloreactive $\mathrm{T}$ cells is the main mechanism maintaining tolerance in a mixed chimera setting [24]. In costimulation blockade-based models, peripheral deletion of donor-reactive CD4/CD8 T cells is also an important contributor to tolerance [24].

Tung and his team have investigated the immunological features of individual components of the murine limb graft, and found that different graft components displayed distinctive immunological features. In particular, skin was the most potent stimulator of $\mathrm{T}$ cell alloimmune responses followed by nerves. Overall, the high immunogenicity of skin in the limb graft in mice is consistent with results obtained from rat studies [25]. In rat VCA, cyclosporine or FK506 monotherapy is sufficient to suppress limb graft rejection as long as the treatment is continued [1]. Similarly in mice, low-dose FK506 monotherapy $(2 \mathrm{mg} / \mathrm{kg} /$ day $)$ significantly prolonged mouse limb graft survival ( $6 \pm 1$ vs. $87 \pm 22$ days) $[7 \bullet \bullet]$.

The first immunomodulatory protocol in mouse VCA was introduced by Tung et al in 2003 [26]. In this study, heterotopic hindlimb transplant was performed between the stringent strain combination of $\mathrm{C} 57 \mathrm{~B} 1 / 6$ and $\mathrm{Balb} / \mathrm{c}$, covered by transient costimulation blockade using anti-CD154 (0.5 mg QOD for 1 week, then weaned off over 2 months). This protocol markedly prolonged limb graft survival $(9.6 \pm 1.1$ vs. $75 \pm 25$ days), but was ineffective in the absence of the bone component (16.2 \pm 2.2 days survival, and no donor chimerism was detected) [26]. In later studies, the authors enhanced their protocol by combining transient $\mathrm{CD} 154$ blockade with CD4/CD8 depletion ( $0.5 \mathrm{mg}$ each QOD for one week), a number of additional treatments including total body 
Table 2 Immune Modulation Strategies in Murine Vascularized Composite Allotransplantation

\begin{tabular}{|c|c|c|c|c|c|}
\hline Author & Year & $\begin{array}{l}\text { Model \& Strain } \\
\text { Combination }\end{array}$ & Modulation Protocol & Chimerism & Graft survival \\
\hline Tung et al [26] & 2003 & $\mathrm{Limb}, \mathrm{C} 57 \mathrm{Bl} / 6 \rightarrow \mathrm{Balb} / \mathrm{c}$ & CD154 block & ND & $40-102$ days \\
\hline Tung et al [27] & 2005 & $\mathrm{Limb}, \mathrm{Balb} / \mathrm{c} \rightarrow \mathrm{C} 57 \mathrm{Bl} / 6$ & CD4/CD8 depletion, CD154 block, donor BM & $<30$ days & $35-91$ days \\
\hline Tung et al [28] & 2005 & $\mathrm{Limb}, \mathrm{C} 57 \mathrm{Bl} / 6 \rightarrow \mathrm{Balb} / \mathrm{c}$ & CD4/CD8 depletion, CD154 block, TBI, donor BM & $\mathrm{N} / \mathrm{A}$ & 28 - 91 days \\
\hline Cohen et al [29] & 2006 & $\mathrm{Limb}, \mathrm{Balb} / \mathrm{c} \rightarrow \mathrm{C} 57 \mathrm{Bl} / 6$ & CD4/CD8 depletion, CD154 block, TBI, donor BM & $2 / 6$ mice at 30 days & $>30$ days \\
\hline Zhong et al [15•] & 2007 & $\mathrm{Limb}, \mathrm{C} 57 \mathrm{Bl} / 6 \rightarrow \mathrm{Balb} / \mathrm{c}$ & CD45RB block, rapamycin, deoxyspergualin analog, & $>100$ days & $>100$ days \\
\hline Li et al $[31 \bullet \bullet]$ & 2008 & $\mathrm{OMC}, \mathrm{Balb} / \mathrm{c} \rightarrow \mathrm{C} 57 \mathrm{Bl} / 6$ & CD4 inhibition, CD154 block, CD8 depletion, rapamycin & $>90$ days & $>200$ days \\
\hline Tung et al [30] & 2008 & $\mathrm{Limb}, \mathrm{Balb} / \mathrm{c} \rightarrow \mathrm{C} 57 \mathrm{Bl} / 6$ & CD28 / CD154 block & ND & $40-139$ days \\
\hline Lin et al $[21 \bullet]$ & 2013 & $\mathrm{OMC}, \mathrm{DBA} / 2 \rightarrow \mathrm{C} 57 \mathrm{Bl} / 6$ & DN Treg, rapamycin, anti-IL2, ALS & $<90$ days & $>180$ days \\
\hline
\end{tabular}

$B M$, bone marrow

$T B I$, total body irradiation

$D N$ Treg, double negative regulatory $\mathrm{T}$ cell

$A L S$, anti-lymphocyte serum

$N D$, not detected

$N / A$, not performed in the study

irradiation (TBI) and/or donor bone marrow infusion (BMT) were added. A transient course of selective $\mathrm{T}$ cell depletion and CD154 blockade using an antibody cocktail only modestly prolonged graft survival (51.4 \pm 17 days) [27], not as significantly as CD154 blockade monotherapy [26]. In addition, combination of the antibody cocktail with TBI and donor BMT only slightly improved graft survival [28], and only two out of six of the mice in the group showed a significant level of mixed chimerism 30 days after transplantation [29]. Interestingly, significant prolongation of graft survival ( $134 \pm 5$ days) was achieved by a transient course of costimulation blockade composed of anti-CD154 and CTLA4-Ig antibodies [30].

Our murine VCA model was introduced in 2007 [19]. In this study, we not only tested the microsurgical feasibility, but also confirmed de novo donor-derived hematopoiesis from the bone graft, with RAG-2 knockout mice as the research tool. In our allogeneic VCA study performed between the most stringent strains $\mathrm{Balb} / \mathrm{c}$ and $\mathrm{C} 57 \mathrm{Bl} / 6$, the tolerance protocol involved a transient course of antibody cocktail composed of CD4 inhibition, CD8 inhibition or depletion and CD154 blockade (1 mg of each monoclonal antibody, days 0 , 2, 4 relative to VCA). Two injections of rapamycin $(12 \mathrm{mg} / \mathrm{kg}$ ) were given when appropriate. In the absence of rapamycin, modest prolongation of graft survival was observed only when CD8 depleting antibody was used in the cocktail, while co-administration of rapamycin and the cocktail achieved indefinite graft survival. However, a secondary skin grafting experiment revealed that robust, donor-specific graft tolerance was only possible when rapamycin was combined with an antibody cocktail containing CD8 depletion [31]. Furthermore, up to $4 \%$ donor mixed chimerism was observed in central and peripheral immune organs accompanying tolerance, and the majority were NK and B cells. We did not observed any signs of graft-versus-host disease in our model [31]. Our approach allowed us to dissect the mechanisms underlying mixed chimerism-associated tolerance. First, we found specific $\mathrm{V} \beta \mathrm{T}$ cell deletion in tolerant mice. Second, we found that thymectomy of recipient mice did not prevent tolerance development. Third, depletion of regulatory $\mathrm{T}$ cells (Treg) in tolerant animals by anti-CD25 antibody abolished robust tolerance in half of the animals. These findings suggested involvement of central and/or peripheral deletion as well as regulatory mechanisms in tolerance associated with mixed chimerism. Both Tung's data and ours provided supportive evidence for the involvement of the CD154 costimulation pathway in the induction of mixed chimerism and tolerance, which is consistent with previous mouse mixed chimerism studies in other types of experimental transplantation models [24].

Our results in the VCA model are consistent with other published evidence supporting a role for Tregs in inducing mixed chimerism and tolerance [24, 31]. In particular, Lin et al performed a Treg-based study in their murine VCA model [21•]. The protocol consisted of rapamycin, an immunosuppressant shown to selectively preserve Tregs; IL-2/Fc fusion protein, a survival factor for Tregs; and adoptive transfer of five million double-negative (DN) Tregs of recipient origin, which was induced to be donor antigen-specific [21•]. DN Treg infusion with rapamycin and IL-2/Fc fusion protein significantly prolonged graft survival, but indefinite graft survival and mixed chimerism was only achieved when being combined with global $\mathrm{T}$ cell depletion. Increased levels of 
Tregs and donor-specific $\mathrm{T}$ cell hyporesponsiveness was observed in the tolerant mice. The authors also noted spontaneous waning of the mixed chimerism starting at 30 days after transplantation, however robust donor-specific tolerance remained at least for 180 days [21•].

The dendritic cell system is believed to play a pivotal role in tolerance induction. Zhong et al introduced their tolerogenic protocol in mouse limb transplantation which appeared to involve dendritic cells [15]. In this study, shortterm combined treatment of anti-CD45RB ( $3 \mathrm{mg} / \mathrm{kg} /$ day $)$ and a 15-deoxyspergualin (DSG) analog called LF 15-0195 (LF, $2 \mathrm{mg} / \mathrm{kg} /$ day) was given to limb graft recipients, with (triple treatments) or without (double treatments) rapamycin $(2 \mathrm{mg} / \mathrm{kg} /$ day). The grafts under double treatments showed significantly prolonged survival ( $9 \pm 1$ vs. $39 \pm 7$ days). On the other hand, seven out of 11 grafts from mice receiving triple treatment had indefinite donor-specific tolerance until the endpoint (100 days) of observation, with low-level mixed chimerism $(1-2 \%)$ maintained throughout the observation period. The underlying mechanism of anti-CD45RB/DSG mediated tolerance was not clear, but their data showed an impaired ability of dendritic cells from tolerant recipients to stimulate $T$ cell proliferation, suggesting a role for dendritic cells in tolerance induction [15].

\section{Conclusion}

Murine VCA models are technically feasible and have obvious advantages for this sector of the field in transplant immunology. However, general adoption and in-depth immunologic analysis of the models has been slowed by the requirement for an extremely high level of microsurgical expertise. For this reason limited information addressing murine VCA immunology has been obtained. Recent introduction of the technically less difficult "cuff" method may help relax this requirement in the future, and facilitate progress in dissecting mechanisms underlying immunological events attending murine VCA.

\begin{abstract}
Acknowledgment The authors thank Dr. Philip M Murphy, Chief of Laboratory of Molecular Immunology, National Institute of Allergy and Infectious Diseases (NIAID), National Institutes of Health, for reading the manuscript and giving constructive comments.
\end{abstract}

\section{Compliance with Ethics Guidelines}

Conflict of Interest Zhanzhuo Li and Alain Le Moine declare that they have no conflict of interest.

Human and Animal Rights and Informed Consent This article does not contain any studies with human or animal subjects performed by any of the authors.

\section{References}

Paper of particular interest, published recently, have been highlighted as:

- of importance

•• of major importance

1. Siemionow M, Klimczak A. Advances in the development of experimental composite tissue transplantation models. Transpl Int. 2010;23(1):2-13.

2. Jensen JN, Mackinnon SE. Composite tissue allotransplantation: A comprehensive review of the literature - Part I. J Reconstr Microsurg. 2000;16(1):57-68.

3. Jensen JN, Mackinnon SE. Composite tissue allotransplantation: A comprehensive review of the literature - Part II. J Reconstr Microsurg. 2000;16(2):141-57.

4. Zhong R. Organ Transplantation in Mice: Current Status and Future Prospects. In: Timmermann W, Gassel HJ, Ulrichs K, Zhong R, Thiede A, (editors). Organ transplantation in Rats and Mice: Microsurgical Techniques and Immunological Principles. Springer; 1998. p. 9-15

5. Brandacher G, Grahammer J, Sucher R, Lee W-PA. Animal models for basic and translational research in reconstructive transplantation. Birth Defects Res C Embryol Today Rev. 2012;96(1):39-50.

6. Lin C-H, Sucher R, Shih Y-C, Ng TW, Mao Q, Lin C-H, et al. The Neck as a Preferred Recipient Site for Vascularized Composite Allotransplantation in the Mouse. Plast Reconstr Surg. 2014;133(2):133e-41.

7.・ Sucher R, Lin C-H, Zanoun R, Atsina K-K, Weinstock M, Pulikkotil B, et al. Mouse hind limb transplantation: a new composite tissue allotransplantation model using nonsuture supermicrosurgery. Transplantation. 2010;90(12):1374-80. This study introduced "cuff" technique into mouse VCA field, and achieved short surgery duration, high success rate and good long-term anastomsis patency.

8. Tung TH, Mohanakumar T, Mackinnon SE. Development of a mouse model for heterotopic limb and composite-tissue transplantation. J Reconstr Microsurg. 2001;17(04):267-74.

9. Cooley BC, Daley R. Free flap transplantation in mice. Microsurgery. 1998;18(5):320-3.

10. Jiang JF, Humar A, Gracia B, Zhong R. Surgical technique for vascularized ear transplantation in mice. Microsurgery. 1998;18(1): 42-6. doi:10.1002/(sici)1098-2752(1998)18:1<42::aid-micr10>3. $0 . \mathrm{co} ; 2$-s.

11. Ulusal AE, Ulusal BG, L-m H, Wei FC. Temporary assisting suspension suture technique for successful microvascular anastomosis of extremely small and thin walled vessels for mice transplantation surgery. Plast Reconstr Surg. 2005;116(5):1438-41.

12. Zhang F, Shi DY, Kryger Z, Moon W, Lineaweaver WC, Buncke HJ. Development of a mouse limb transplantation model. Microsurgery. 1999;19(5):209-13.

13. Foster RD, Liu T, Wu J. Orthotopic hindlimb transplantation in the mouse. J Reconstr Microsurg. 2003;19(01):049-52.

14. Zimmermann FA, Butcher GW, Davies HS, Brons G, Kamada N, Turel O. Techniques for Orthotopic Liver Transplantation in the Rat and Some Studies of the Immunological Responses to Fully Allogeneic Liver Grafts. Transplant Proc. 1979;11(1):571-7.

15. Zhong T, Liu Y, Jiang J, Wang H, Temple CL, Sun H, et al. Longterm limb allograft survival using a short course of anti-CD45RB monoclonal antibody, LF 15-0195, and rapamycin in a mouse model. Transplantation. 2007;84(12):1636-43.

16. Tung TH, Mohanakumar T, Mackinnon SE. A subcutaneous heterotopic limb transplantation model in the mouse for prolonged allograft survival. Microsurgery. 2001;21(7):298-305. 
17. Tung TH, Mohanakumar T, Mackinnon SE. Effect of age on thrombosis and morbidity in the mouse-limb transplantation model. J Reconstr Microsurg. 2002;18(01):047-52.

18. Yan Y, Johnson PJ, Glaus SW, Hunter DA, Mackinnon SE, Tung TH. A novel model for evaluating nerve regeneration in the composite tissue transplant: the murine heterotopic limb transplant. Hand. 2011;6(3):304-12.

19. Li Z, Benghiat FS, Kubjak C, Schuind F, Goldman M, Le Moine A. Donor T-cell development in host thymus after heterotopic limb transplantation in mice. Transplantation. 2007;83(6):815-8.

20. Green C SS. Basic Vascular Exercises. Basic Microsurgical Techniques: A Laboratory Manual. Sandoz Ltd. \& Carl Zeiss Ltd. p. 26.

21. Lin C, Zhang W, Ng T, Zhang D, Jiang J, Pulikkottil B, et al. Vascularized Osteomyocutaneous Allografts Are Permissive to Tolerance by Induction-Based Immunomodulatory Therapy. Am J Transplant. 2013;13(8):2161-8. This study provides evidence for the involvement of donor-specific regulatory T cells in the induction of donor-specific tolerance in mouse VCA.

22. Sucher R, Lin C-H, Oberhuber R, Kern B, Zheng XX, Zelger B, et al. Hemiface allotransplantation in the mouse. Plast Reconstr Surg. 2012;129(4):867-70.

23. Shipchandler TZ, Lott DG, Lorenz RR, Friedman AD, Dan O, Strome M. New mouse model for studying laryngeal transplantation. Ann Otol Rhinol Laryngol. 2009;118(6):465.

24. Pilat N, Hock K, Wekerle T. Mixed chimerism through donor bone marrow transplantation: a tolerogenic cell therapy for application in organ transplantation. Curr Opin Organ Transplant. 2012;17(1):6370. doi:10.1097/MOT.0b013e32834ee68b.

25. Tung TH, Mohanakumar T, Mackinnon SE. TH1/TH2 cytokine profile of the immune response in limb component transplantation. Plast Reconstr Surg. 2005;116(2):557-66.

26. Tung TH, Mackinnon SE, Mohanakumar T. Long-term limb allograft survival using anti-CD40L antibody in a murine model. Transplantation. 2003;75(5):644-50.

27. Tung TH, Mackinnon SE, Mohanakumar T. Prolonged limb allograft survival with CD40 costimulation blockade, T-cell depletion, and megadose donor bone-marrow transfusion. Microsurgery. 2005;25(8):624-31.

28. Tung TH, Mackinnon SE, Mohanakumar T. Combined treatment with CD40 costimulation blockade, T-cell depletion, low-dose irradiation, and donor bone marrow transfusion in limb allograft survival. Ann Plast Surg. 2005;55(5):512-8.

29. Cohen M, Mohanakumar T, Mackinnon SE, Tung TH. Chimerism after vascularized limb versus bone marrow transplantation. J Reconstr Microsurg. 2006;22(05):375-84.

30. Tung T, Mackinnon S, Mohanakumar T. Costimulation blockade of CD40 and CD28 pathways in limb transplantation. Transplantation proceedings. 2008;40(10):3723-4.

31. Li Z, Benghiat F, Charbonnier LM, Kubjak C, Rivas MN, Cobbold S, et al. CD8+ T-Cell Depletion and Rapamycin Synergize with Combined Coreceptor/Stimulation Blockade to Induce Robust Limb Allograft Tolerance in Mice. Am J Transplant. 2008;8(12): 2527-36. 